\title{
The first CCD photometric study of the open cluster NGC 2126
}

\author{
A. Gáspár ${ }^{1,5}$, L. L. Kiss ${ }^{2,5, \star}$, T. R. Bedding ${ }^{2}$, A. Derekas ${ }^{2,5}$, S. Kaspi ${ }^{6}$, Cs. Kiss ${ }^{4}$, K. Sárneczky 3,5 , \\ Gy. M. Szabó ${ }^{1}$, and M. Váradi ${ }^{1,5}$ \\ ${ }^{1}$ Department of Experimental Physics and Astronomical Observatory, University of Szeged, Szeged, Dóm Tér 9, \\ 6720, Hungary \\ 2 School of Physics, University of Sydney 2006, Australia \\ 3 Astronomical Observatory, Szeged, Hungary \\ ${ }^{4}$ Konkoly Observatory of the Hungarian Academy of Sciences, PO Box 67, 1525 Budapest, Hungary \\ 5 Guest Observer at Konkoly Observatory \\ ${ }^{6}$ School of Physics and Astronomy and the Wise Observatory, Tel-Aviv University, Tel-Aviv 69978, Israel
}

Received 1 April 2003 / Accepted 18 August 2003

\begin{abstract}
We present the first CCD photometric observations of the northern open cluster NGC 2126. Data were taken on eight nights in February and December 2002 with a total time span of $\sim 57$ hours. Almost 1000 individual $V$-band frames were examined to find short-period variable stars. We discovered six new variable stars, of which one is a promising candidate for an eclipsing binary with a pulsating component. Two stars were classified as $\delta$ Scuti stars and one as Algol-type eclipsing binary. Two stars are slow variables with ambiguous classification. From absolute $V(R I)_{\mathrm{C}}$ photometry we have estimated the main characteristics of the cluster: $m-M=11^{\mathrm{m}} \cdot 0 \pm 0 . \mathrm{m}, E(V-I)=0^{\mathrm{m}} \cdot 4 \pm 0^{\mathrm{m}} \cdot 1, E(V-R)=0^{\mathrm{m}} \cdot 08 \pm 0^{\mathrm{m}} \cdot 06\left(E(B-V)=0^{\mathrm{m}} \cdot 2 \pm 0^{\mathrm{m}} \cdot 15\right)$ and $d=1.3 \pm 0.6 \mathrm{kpc}$. Cluster membership is suggested for three variable stars from their positions on the colour-magnitude diagram.
\end{abstract}

Key words. open cluster and associations: general - open clusters and associations: individual: NGC 2126 stars: variables: general $-\delta$ Sct

\section{Introduction}

Variable stars in clusters are crucial tools of stellar astrophysics: the application of stellar evolutionary theories via isochrone fitting of the colour-magnitude diagrams yields temperature, luminosity and age values for the member stars, which in turn draw strong constraints on pulsational properties (e.g. Frandsen \& Arentoft 1998) or binary evolution (Rucinski et al. 1996). Early research of $\delta$ Scuti pulsation in open clusters (Breger 1972) and its revival with the wide application of the CCD technique (for a review of the current state see Rodríguez \& Breger 2001) have recently been extended toward the new class of $\gamma$ Dor variables (Handler 1999), which have been found in a number of young and intermediate-age open clusters (Arentoft et al. 2001; Kim et al. 2001; Choo et al. 2003 and references therein). Clusters of different parameters can help map out the dependence of pulsational properties on age and metallicity, thus allowing better understanding of physical mechanisms driving the pulsation. On the other hand, detached eclipsing double-lined spectroscopic binaries can serve as very accurate distance indicators (e.g. Thompson et al. 2001), with the potential of feeding back to the isochrone fitting method

Send offprint requests to: L. L. Kiss,

e-mail: laszlo@physics.usyd.edu.au

* On leave from University of Szeged, Hungary. itself. This work aims to contribute to these issues with the first CCD observations of the northern open cluster NGC 2126, with particular emphasis on its variable star content.

NGC $2126\left(=\right.$ C0559+499, $\alpha_{2000}=06^{\mathrm{h}} 02^{\mathrm{m}} \cdot 55, \delta_{2000}=$ $+49^{\circ} 52^{\prime}$, Trümpler class III $2 \mathrm{~m}: \mathrm{b}$ ) is a moderately rich typical galactic cluster with several dozens of members scattered in a region of 5-6' (Lyngå 1987) in the constellation Auriga. The only previous photometric study was presented by Cuffey (1943), who made blue and red ( $\lambda \lambda$ 4300-6200) photographic observations. He estimated the distance to NGC 2126 as $950 \mathrm{pc}$, based on a comparison to M 35. The WEBDA cat$\operatorname{alog}^{1}$ reflects this lack of data, as no other source is listed for NGC 2126. This neglect turned our attention toward NGC 2126 and the present study presents an analysis of 8 nights of observations obtained in 2002 . The measurements and data reduction are described in Sect. 2. Cluster parameters and the six new variable stars are discussed in Sect. 3, and a brief summary is given in Sect. 4.

\section{Observations and data reduction}

CCD $V(R I)_{\mathrm{C}}$ observations were carried out on five consecutive nights in February 2002 and three nights in December 2002.

\footnotetext{
${ }^{1}$ http://obswww.unige.ch/webda
} 
Table 1. The journal of observations.

\begin{tabular}{lcccc}
\hline \hline Date & $\begin{array}{c}V \\
\text { frames }\end{array}$ & $\begin{array}{c}R_{\mathrm{C}} \\
\text { frames }\end{array}$ & $\begin{array}{c}I_{\mathrm{C}} \\
\text { frames }\end{array}$ & $\begin{array}{c}\text { length } \\
\text { (hours) }\end{array}$ \\
\hline 2002 Feb. 1/2 & 83 & 2 & 3 & 8.5 \\
2002 Feb. 2/3 & 108 & 1 & 1 & 8.8 \\
2002 Feb. 3/4 & 142 & - & - & 8.7 \\
2002 Feb. 4/5 & 134 & - & - & 8.7 \\
2002 Feb. 5/6 & 129 & - & - & 8.7 \\
2002 Dec. 23/24 & 62 & - & - & 3.4 \\
2002 Dec. 25/26 & 215 & - & - & 5 \\
2002 Dec. 31/01 & 101 & - & - & 5 \\
\hline Total: 8 nights & 974 & 3 & 4 & 56.8 \\
\hline
\end{tabular}

We used the 60/90/180 $\mathrm{cm}$ Schmidt telescope mounted at the Piszkéstetô Station of the Konkoly Observatory. The detector was a Photometrics AT-200 CCD camera (1536× 1024 pixels, KAF-1600 chip with UV-coating). The image scale was $1^{\prime \prime}$. $1 /$ pixel, giving a $29^{\prime} \times 18^{\prime}$ field of view. The observations consisted of $V$-band time-series observations (mostly $180 \mathrm{~s}$ exposures) and calibrated $V(R I)_{\mathrm{C}}$ photometric observations (from $60 \mathrm{~s}$ to $600 \mathrm{~s}$ exposures). The full observing $\log$ is presented in Table 1. The observed field and the new variable stars are shown in Fig. 1.

The image reduction was done with standard tasks in $\mathrm{IRAF}^{2}$. For flat field corrections, we used sky flat images taken during the evening and morning twilights. We performed psf-photometry with the daophot package in IRAF using the Moffatt point-spread function, which was found to be wellsuited for fitting the slightly distorted stellar profiles of the used instrument (Kiss et al. 2001).

For photometric calibrations, we observed standard stars in the open cluster M 67 from the list of Chevalier \& Ilovaisky (1991). The instrumental magnitudes were transformed with the photcal package, according to the following standard transformation equations:

$$
\begin{aligned}
& V=v-0.136 X+0.126(V-R)-5.180, \sigma=0.03 \\
& (V-I)=1.028((v-i)-0.079 X)+0.348, \sigma=0 .{ }^{\mathrm{m}} 02 \\
& (V-R)=0.827((v-r)-0.034 X)-0.007, \sigma=0 .{ }^{\mathrm{m}} 04
\end{aligned}
$$

where the symbols have their usual meanings. For the calibration we used 19 stars of the 29 listed by Chevalier \& Ilovaisky (1991). The colour ranges were $-0.03 \ldots 0.64$ and -0 m $07 \ldots 1$. 21 for $V-R$ and $V-I$, respectively, so that tranformations of the reddest stars in the field (about $10 \%$ of the sample) were extrapolations. Consequently, there might be some systematic distorsion in the reddest part of the colourmagnitude diagram.

The transformation of the cluster was done in two steps. First, we calibrated non-saturated stars on three images (one for each filter) confined by consecutive M 67 observations on

\footnotetext{
${ }^{2}$ IRAF is distributed by the National Optical Astronomy Observatories, which are operated by the Association of Universities for Research in Astronomy, Inc., under cooperative agreement with the National Science Foundation.
}

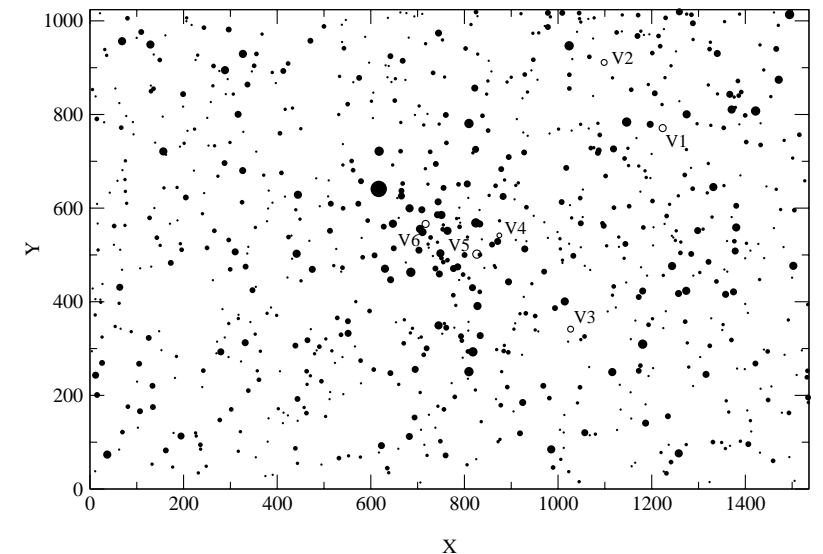

Fig. 1. The observed CCD field $\left(29^{\prime} \times 18^{\prime}\right)$ of the open cluster NGC 2126. The new variable stars are shown with open circles. Symbol sizes are proportional to the stellar brightnesses. The largest concentration of stars is about $8^{\prime}$ in diameter, centred at $(750,550)$. North is up, east is to the left.

February 2, 2002. Then we co-added a number of $V$ band images and all $(R I)_{\mathrm{C}}$ frames and measured fainter stars relative to previously calibrated local standards. We estimate the final photometric error as \pm 0 . 05 , which is likely to be an overestimate for the brightest stars.

Time-series data were reduced in two separate subsets ( 5 nights in February and 3 nights in December), because the follow-up observations in December, 2002 were obtained under a different instrumental setup (the CCD imager was rotated by $90^{\circ}$, so that a certain field of view was excluded from the measurements). Within the subsets, CCD frames were matched by the task imalign. Since the cluster is not too crowded, instrumental magnitudes could be easily identified based on the $X-Y$ pixel coordinates. Stars lying closer than 30 pixels to the image edge were rejected. Differential light curves were calculated with the ensemble normalization technique (Gilliland \& Brown 1988), for which dozens of stars of medium brightness were selected. We could identify almost 800 stars in the February run and that was the light curve dataset in which we searched for variable stars. The search was performed by checking the light curve statistics and by visual inspection of the suspect time-series data. We calculated the scatter of individual light curves for every star and plotted it against the apparent brightness. Outstanding points referred to either variable stars or constant stars with psf-fitting difficulties (e.g. crowding). In this way we found six stars to be intrinsic variables. In December, 2002, we focused on these six variables and the ensemble comparison stars, so that it is possible that there are unnoticed slow and/or long-period variables in the cluster field.

The light curves were further analysed to search for possible periodicities. For this, we calculated Fourier spectra with Period98 (Sperl 1998). For the eclipsing binaries, the phase dispersion minimisation (PDM, Stellingwerf 1978) provided a better period searching method. Our original data are available electronically at the CDS via anonymous ftp to cdsarc.u-strasbg.fr (130.79.128.5) or via http:// cdsweb.u-strasbg.fr/cgi-bin/qcat?J/A+A/410/879 

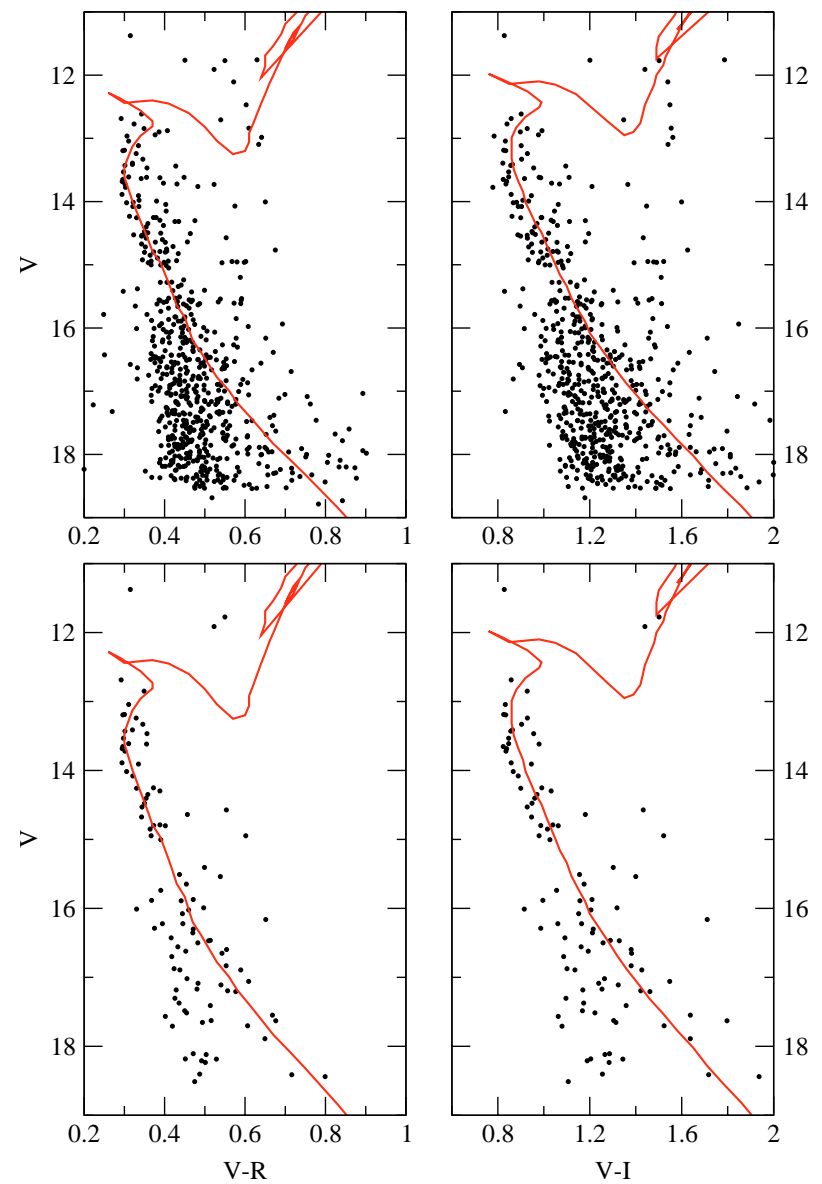

Fig. 2. The colour-magnitude diagrams of NGC 2126. Top row: full field of view; bottom row: the central field of $8^{\prime}$ diameter. The solid lines show the $\log t=9.1$ isochrone.

\section{Results}

\subsection{The colour-magnitude diagram}

The physical parameters of the cluster were estimated by fitting isochrones to the colour-magnitude diagram (CMD). In order to separate the cluster's stars from other field stars, we have examined proper motions taken from the USNO B1.0 catalog (Monet et al. 2003). We found that of the 800 stars, almost 200 have proper motions detected in the USNO plate material. However, those stars are evenly distributed over the field with no concentration around the cluster, so that NGC 2126 turned out to be in the background. Consequently, we removed all stars from the CMD with non-zero proper motions and the resulting CMDs are shown in the top row of Fig. 2. This procedure has been checked by two nearby galactic fields, offset by about 1 degree north and south. A comparison of proper motion histograms showed that we have indeed selected the galactic foreground.

In order to decrease background contamination, we kept only the inner $8^{\prime}$ of the cluster for the isochrone fitting (bottom row in Fig. 2). This region contains 103 stars between $V=11^{\mathrm{m}} 3-18.5$, of which the majority seems to be easily distinguishable from the background and some weak foreground. We assumed solar composition and included reddening determination in the fitting procedure. The isochrones were taken from Bertelli et al. (1994) and shifted individually to match the main sequence, turn-off point and red giant positions. We found that the overall shape of the CMD is well reproduced with isochrones of $\log t$ ranging from 9.0 to 9.3 , $E(V-I)$ between 0.53 and $0.33, E(V-R)$ between 0.14 and 0.03 and distance moduli between 11.5 and 10.4 . We plot the $\log t=9.1$ isochrone in Fig. 2, showing the "best" fit, but the difference for the other isochrones is almost negligible. Changing the composition makes the parameter ranges even wider, thus the estimated parameter errors are quite large and a further spectroscopic study addressing spectral types and chemical composition is desirable.

In this paper we adopt the following physical parameters: $m-M=11^{\mathrm{m}} 0 \pm 0 . \mathrm{m} 6, E(V-I)=0{ }^{\mathrm{m}} \cdot 4 \pm 0 . \mathrm{m} 1, E(V-R)=$ $0.08 \pm 0$. 06 . The reddenings were converted to $E(B-V)$ using the coefficients listed in Rieke \& Lebofsky (1985): $E(V-$ $R) / E(B-V)=0.78, E(V-I) / E(B-V)=1.60$. The result is $E(B-V)=0 . \mathrm{m} 2 \pm 0 . \mathrm{m} 15$, and the distance to the cluster (assuming $\left.A_{V} \approx 3.1 \times E(B-V)\right)$ is $1.3 \pm 0.6 \mathrm{kpc}$.

\subsection{New variable stars}

Nightly light curves of the new variable stars are shown in Fig. 3 (numbered in order of increasing right ascension). Differential light curve data were shifted so that the mean values match the apparent magnitudes of the stars in the CMD. Therefore, the absolute $V$ magnitudes in Fig. 3 are somewhat uncertain, depending on the magnitude range of the variables.

The light curve shapes suggest the following classifications: V1 and V2 are slow variables of ambiguous nature; V3 and V5 are short-period variables, most likely of $\delta$ Scuti type; V4 is an Algol-type eclipsing binary with only one observed minimum, while the light curve of V6 is a mixture of $\delta$ Scutilike oscillations and Algol-like eclipses.

\subsubsection{V1 and V2: Slow variations}

Although these two stars showed clear variability, our dataset is too short to determine reliable periods. For V1, both PDM and Fourier analysis suggested $P=1.64470 \mathrm{~d}$ or half that value, $0.82235 \mathrm{~d}$ (the uncertainties are a few in the last digit). We plot the resulting phase diagrams in Fig. 4 (the epoch of maximum is HJD 2452307.48 ).

With no colour light curve, the classification is uncertain. For $0.822235 \mathrm{~d}$, one can speculate on possible $\gamma$ Dor-like oscillations. These stars are early F-type mainsequence or subgiant stars with periods $0.4-3.0 \mathrm{~d}$ and $V$ band amplitudes less than $0 . \mathrm{m} 1$, pulsating in non-radial gravity modes. According to the definition by Handler (1999), the $\gamma$ Dor instability strip covers the $7200-7700 \mathrm{~K}$ range on the zero-age main sequence (ZAMS) and the 6900-7500 $\mathrm{K}$ range one magnitude above the ZAMS. Assuming cluster membership, the dereddened colours of V1 are $(V-$ $R)_{0}=0.24$ and $(V-I)_{0}=0.48$. Standard tabulations give an effective temperature of 6500-7000 K (Cox 2000), which marginally supports the classification as a $\gamma$ Dor star. Also, the cluster membership would imply an absolute 


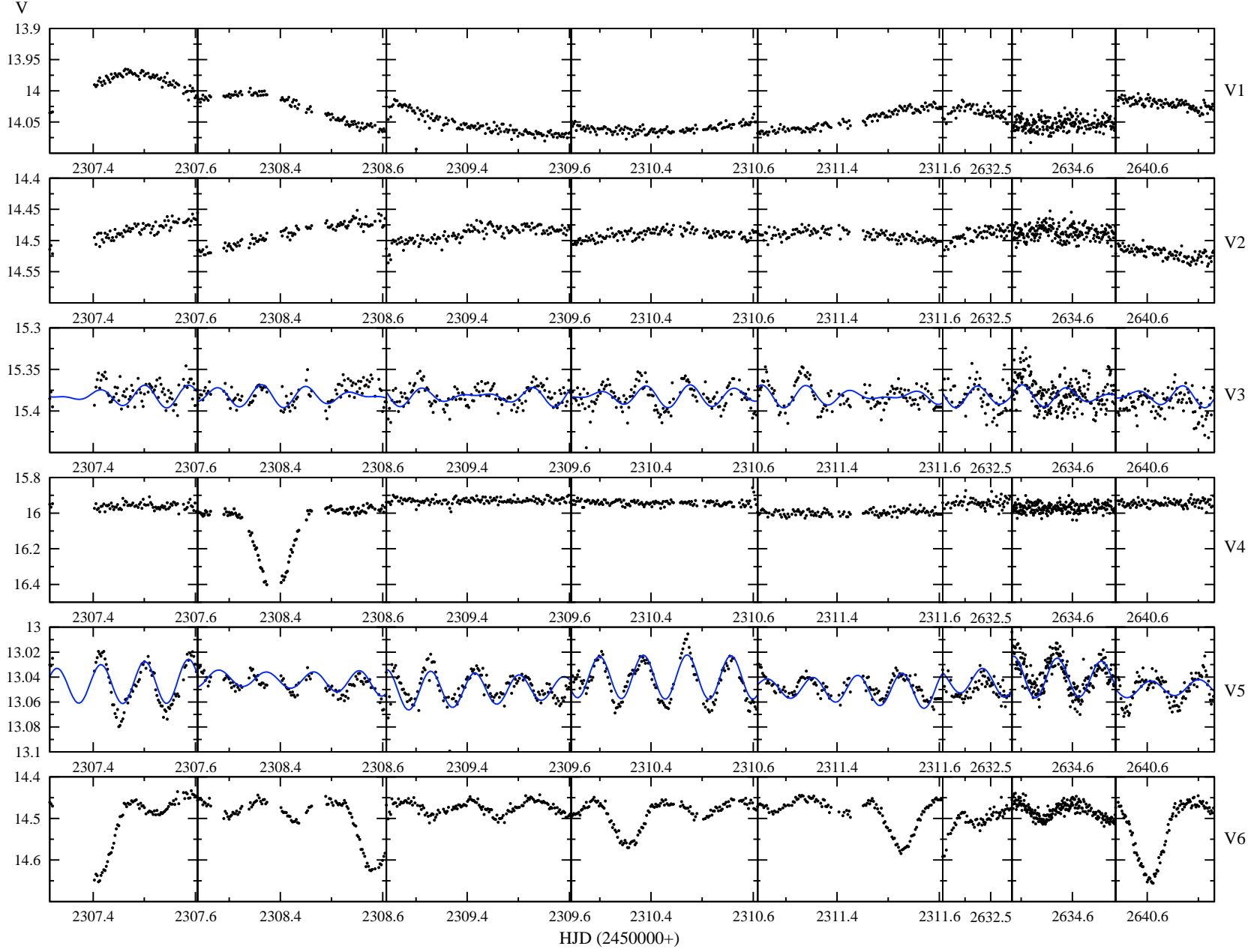

Fig. 3. Individual light curves of the six new variable stars. The solid lines denote Fourier fits for V3 and V5.

magnitude $M_{V}=3$. 0 ; the temperature and absolute magnitudes are consistent with the coolest $\gamma$ Dor variables. In this case, the apparent amplitude modulation is due to the presence of other excited modes of pulsation and a proper asteroseismological analysis would require a much longer dataset.

On the other hand, for $P=1.6447 \mathrm{~d}$, the double-peaked light curve is similar to those observed for spotted active binaries of RS CVn subtype. For instance, recent data collection of Cutispoto et al. (2003) contains nice examples of very similar double-peaked curves of well-known active stars (BI Cet, $\mathrm{BC}$ Phe). Based on the present dataset, it is impossible to distinguish between the two classifications. The fact that V1 lies quite far from the cluster centre (about 10') weakens the possibility of membership and consequently makes the temperature and absolute magnitude estimates less reliable.

For V2, the data can be equally well folded with periods around $0.5 \mathrm{~d}$ and $1 \mathrm{~d}$, but neither period gives a continuous phase diagram with no gap. Therefore, we cannot decide the cause of the low-amplitude variability of this star (the total range is about 0.08 ).

\subsubsection{V4: An Algol-type binary}

We observed only one minimum of the star (at HJD 2452308.387), so that no firm conclusion can be
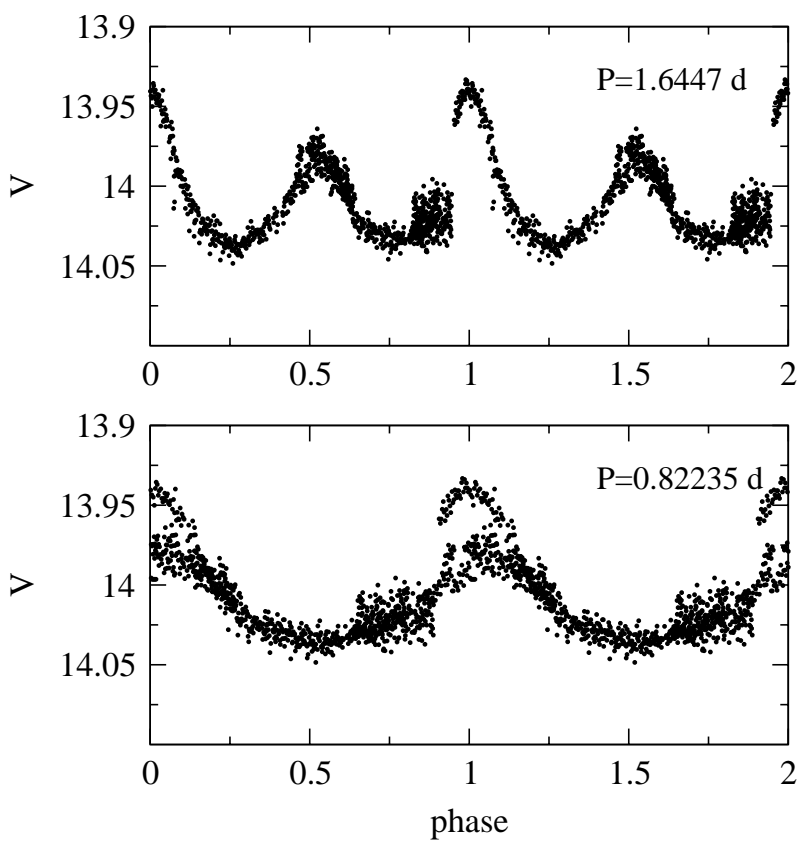

Fig. 4. Phase diagrams for V1 with two equally possible periods.

drawn on its period. However, if we plot only the February subset in one light curve (Fig. 5), the shape suggests a clearly 


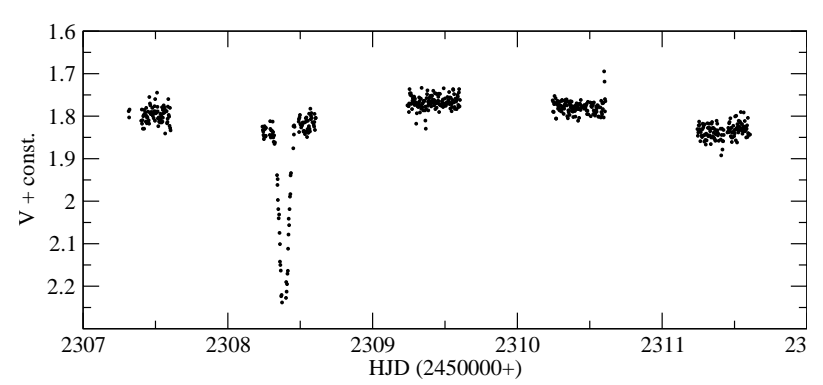

Fig. 5. The 2002 February data for V4.
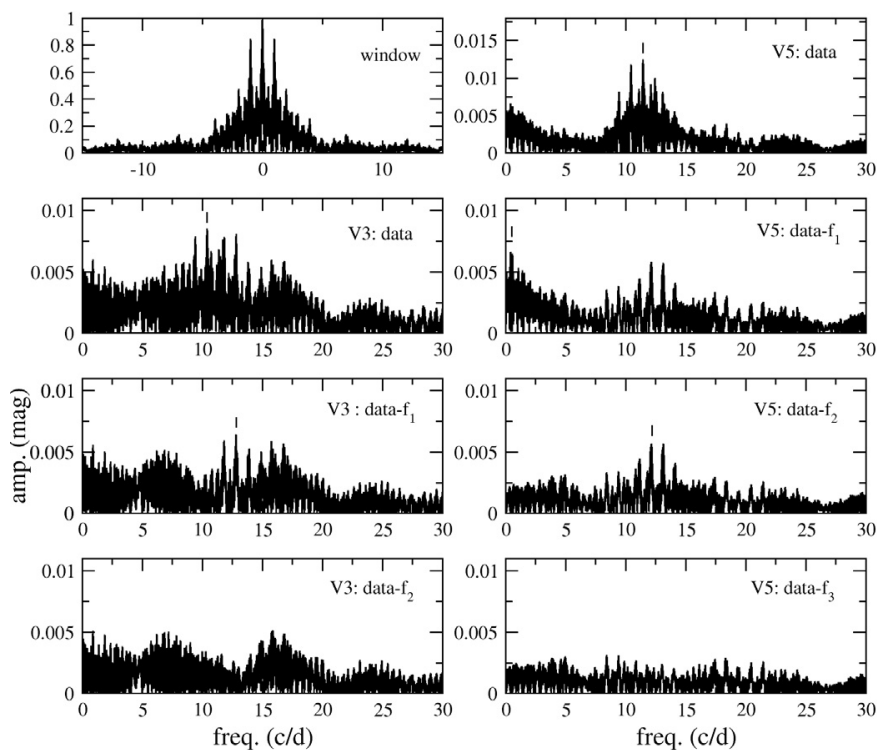

Fig. 6. Fourier analysis of V3 and V5.

visible reflection effect (about $0 .{ }^{\mathrm{m}} 1$ ). Assuming symmetry around the unobserved secondary minimum, a tentative period of $\sim 3$ days can be estimated (i.e. the next primary minimum happened immediately before or after the last night of observations in February).

\subsubsection{V3 and V5: Multiply periodic oscillations}

Both stars showed rapid oscillations with full amplitudes of a few tens of millimags, characteristic of $\delta$ Scuti variables. We have determined the pulsational frequencies with Fourier analysis consisting of iterative prewhitening steps (see Fig. 6).

We could identify two frequencies in both light curves (see Table 2) with $S / N$ ratios (Breger et al. 1993) larger than 4. For V5, the low-frequency component refers to slight night-tonight variations of the mean brightness level, which we suspect were caused by a closely separated field star (see Fig. 1) lying $\sim 7$ " from V5. It is fainter by about 4 mag, so that it may affect profile fitting to some small extent. Nightly variations of the seeing may also introduce variable contribution from this star.

In summary, V3 and V5 are probably multiply periodic pulsating $\delta$ Scuti variables. The frequency ratios (V3: $f_{1} / f_{2}=0.81$, V5: $\left.f_{1} / f_{2}=0.94\right)$ suggest non-radial modes of pulsation for both stars. Small deviations from the light curve fits (Fig. 3) and residual structures of the prewhitened spectra (especially for V3) suggest the probable existence of more
Table 2. The results of the period analyses.

\begin{tabular}{lrrrr}
\hline \hline & $i$ & $f_{\mathrm{i}}\left(\mathrm{d}^{-1}\right)$ & amp. (mmag) & $S / N$ \\
\hline V3 & & & & \\
& 1 & 10.39 & 7.6 & 5 \\
& 2 & 12.79 & 6.5 & 4.3 \\
\hline V5 & & & & \\
& 1 & 11.43 & 11.9 & 12 \\
& 2 & 0.42 & 5.3 & 3.8 \\
& 3 & 12.14 & 5.9 & 6 \\
\hline
\end{tabular}

frequencies. Assuming their cluster membership implies the following dereddened colours and absolute magnitudes: V3 $(V-R)_{0}=0.25,(V-I)_{0}=0.5, M_{V} \approx 4.4 ;$ V $5-$ $(V-R)_{0}=0.23,(V-I)_{0}=0.43, M_{V} \approx 2^{\mathrm{m}}$. 0 . The latter values are consistent with typical parameters of $\delta$ Sct stars (Rodríguez $\&$ Breger 2001), we therefore conclude V5 is likely to be a member of the cluster. Moreover, its proximity to the cluster centre supports this conclusion. On the other hand, V3 seems to be a background object, since its absolute magnitude, assuming cluster membership, places it well below the main sequence.

\subsubsection{V6: An eclipsing binary with a pulsating component?}

The most intriguing star is V6, which shows complex light variations. We observed well-defined minima - three deeper and two shallower - characteristic of an eclipsing binary with components of different temperatures. Moreover, the star showed steady oscillations outside eclipses, with amplitude and cycle length characteristic of $\delta$ Scuti-like pulsation (about 0.05 and $0.13 \mathrm{~d}$ ). The symmetric stellar profile, even on the best CCD images, excluded the possibility of two unresolved stars, so that we conclude V6 is an eclipsing binary with at least one pulsating component. Such systems are spectacular targets for asteroseismology, because independent determination of the physical parameters (mass, radius, temperature), applying binary star astrophysics, gives strong constraints on the possible mode identification (e.g. Mkrtichian et al. 2002; Kim et al. 2002a). In addition, eclipses of a pulsating star give spatial resolution across the stellar surface and thereby provide the possibility for mode identification (e.g. Reed et al. 2002).

The period analysis resulted in $P_{\text {orb }}=1.17320$ (3) d. The data were folded with this period and the phase diagram is shown in Fig. 7. The depth is 0.2 and 0.12 for the primary and secondary minima, respectively. The oscillations are clearly visible outside minima and we note that the scatter of the phase diagram after folding only the February data was fairly small. This indicated that the oscillations were quite coherent within 5 consecutive nights in February. Their amplitude was about 0.05 associated with a period $P_{\text {pul }} \approx 0.13 \mathrm{~d}$.

We also performed a separate period analysis of data from which eclipse minima were excluded. This showed the oscillations to be surprisingly stable. They have a period $P_{\text {pul }}=0.12936 \mathrm{~d}$ and the phase diagram shows remarkable small scatter (Fig. 8). The subsequent prewhitening steps indicated a low-frequency component, approximately 


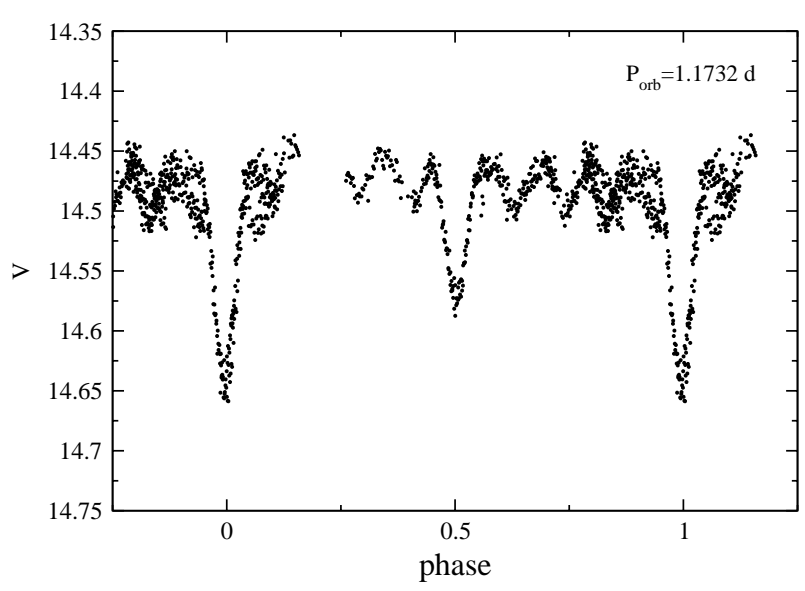

Fig. 7. Phase diagram for V6.

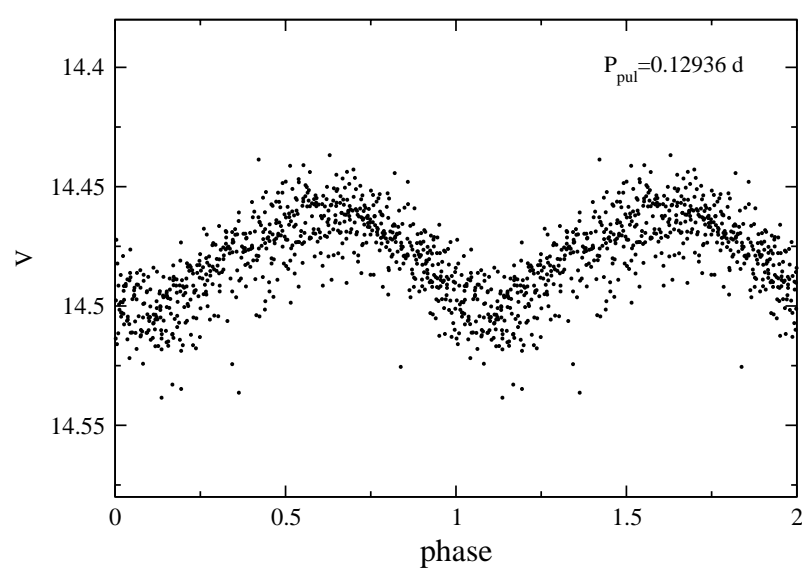

Fig. 8. Outside-eclipse data folded with $P_{\text {pul }}=0.12936$ d. The zero phase corresponds to a primary eclipse.

$2 / T_{\text {obs }}$, and $f_{3} \approx f_{\text {pul }}-f_{\text {orb }}$. Another interesting result is that $f_{\text {pul }} / f_{\text {orb }}=9.07$, suggesting that there might be a $1: 9$ resonance between the orbital motion and pulsation.

We have also observed V6 with the $40^{\prime \prime}$ telescope at the Tel-Aviv University Wise Observatory on 2003 April 10. The spectrograph $10^{\prime \prime}$ long-slit was aligned at $\mathrm{PA}=133.8^{\circ}$ in order to maximize the separation between the V6 spectrum and the nearby objects residing south-east to it. In this way, we minimized the possibility of light from the nearby objects contaminating the V6 spectrum. The spectrum shows prominent hydrogen absorption lines accompanied by the sodium D-doublet (Fig. 9). The spectral lines and the continuum shape suggest an F-type star, typical for a $\delta$ Scuti star, so that the oscillations may be attributed to $\delta$ Scuti-like pulsations.

Out of the six variables, only V6 has non-zero proper motion $\left(\mu_{\alpha} \cos \delta=-14\right.$ mas/year, $\mu_{\delta}=10$ mas/year), thus cluster membership can be excluded.

In the literature, only a few similar system are reported, making V6 a very interesting variable star. Rodríguez \& Breger (2001) listed nine $\delta$ Scuti variables in eclipsing binaries, of which RZ Cas (Ohshima et al. 2001) and AB Cas (Rodríguez et al. 1998) are the best studied. Compared to the known examples, V6 is different in two important respects: i) the relative amplitude ratio $\left(\Delta V_{\text {prim }} / \Delta V_{\text {pul }} \approx 4\right)$ is much smaller than that

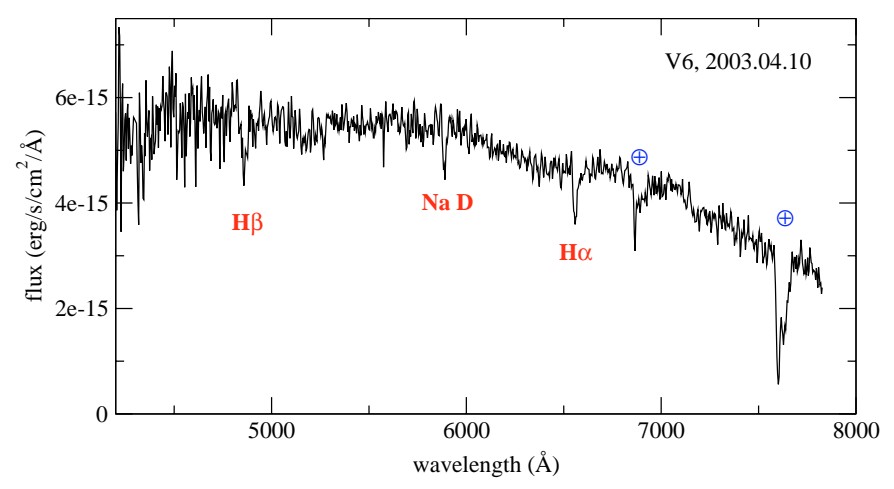

Fig. 9. A low-resolution spectrum obtained on April 10, 2003.

of any other similar stars (the next is V577 Oph, with a ratio of 13$)$; ii) the period ratio $\left(P_{\mathrm{orb}} / P_{\mathrm{pul}} \approx 9\right)$ is much smaller than usual - from Table 3 in Rodríguez \& Breger (2001), only WX Eri has a ratio smaller than 20 (5.005).

Very recently, several new examples of pulsating components in eclipsing binaries have been found (RX Hya, Kim et al. 2002b, 2003; AB Per, Kim et al. 2002c, 2003; TW Dra, Kim et al. 2003; AS Eri, Gamarova et al. 2000), but all are typical low-amplitude rapid pulsators not resembling V6. In that sense, V6 is a unique candidate for an eclipsing binary with a pulsating component. Future spectrophotometric observations, accompanied by radial velocity measurements, are needed to settle a number of questions regarding V6. In particular, are the cyclic changes caused by stellar pulsation? Which component is pulsating? What is the true amplitude of the oscillations, after removing the second light from the light curve (could this be the first high-amplitude $\delta$ Scuti star in an eclipsing binary)? Is there indeed resonance between orbital motion and pulsation? If so, are the oscillations induced by tidal forces (Willem \& Aerts 2002)?

\section{Conclusions}

This paper presents the first CCD photometric study of the northern open cluster NGC 2126 . The $V(R I)_{C}$ observations revealed the main characteristics of the cluster and led to the discovery of six new variable stars. The locations of the variables on the CMD (Fig. 10) suggest membership for V1, V4 and V5; $\mathrm{V} 2$ and V3 are most likely non-member stars, while V6 is a foreground object. The most important results of this research are the following:

1. We have estimated important physical parameters of the cluster with standard photometric methods for the first time.

2. We have discovered six variable stars in a field of $29^{\prime} \times 18^{\prime}$ centred on the cluster, which is about $8^{\prime}$ in diameter. Two are pulsating stars, one is an eclipsing binary with unknown period, one showed a combined light variation of eclipses and pulsations, while two stars have ambiguous classification.

3. The most interesting variable star is V6, which is a promising candidate for an eclipsing binary with a pulsating 
Table 3. The basic data of new variable stars. Except V6, coordinates and identifications were taken from the Guide Star Catalog, Version 2.2 (STSci 2001).

\begin{tabular}{|c|c|c|c|c|c|c|c|c|c|c|}
\hline- & GSC2.2 number & $\alpha_{2000}$ & $\delta_{2000}$ & $\bar{V}$ & $(V-R)$ & $(V-I)$ & $P\left[{ }^{\mathrm{d}}\right]$ & $\Delta V$ & Epoch & Type \\
\hline $\mathrm{V} 1$ & N2110020273 & $6^{\mathrm{h}} 01^{\mathrm{m}} 44.32^{\mathrm{s}}$ & $+49^{\circ} 56^{\prime} 32.5^{\prime \prime}$ & 14.01 & $0^{\mathrm{m}} 32$ & 0.98 & 1.6447 or 0.82235 & 0.12 & 2452307.47 & GDOR? \\
\hline $\mathrm{V} 2$ & N21100206826 & $6^{\mathrm{h}} 01^{\mathrm{m}} 57.68^{\mathrm{s}}$ & $+49^{\circ} 58^{\prime} 56.5^{\prime \prime}$ & 14.52 & 0.32 & 0.88 & - & 0.08 & - & - \\
\hline $\mathrm{V} 3$ & N21100204078 & $6^{\mathrm{h}} 02^{\mathrm{m}} 05,49^{\mathrm{s}}$ & $+49^{\circ} 49^{\prime} 14,5^{\prime \prime}$ & $15 \mathrm{~m} \cdot 38$ & $0^{\mathrm{m}} 33$ & 0.90 & $0.096,0.078$ & 0.08 & - & DSCT \\
\hline V4 & N21100204944 & $6^{\mathrm{h}} 02^{\mathrm{m}} 21,25^{\mathrm{s}}$ & $+49^{\circ} 52^{\prime} 38,4^{\prime \prime}$ & $15 \cdot 99$ & 0.49 & $1 . \mathrm{m} 31$ & - & 0.40 & 2452308.387 & EA \\
\hline V5 & N2110020321 & $6^{\mathrm{h}} 02^{\mathrm{m}} 26,67^{\mathrm{s}}$ & $+49^{\circ} 51^{\prime} 55,2^{\prime \prime}$ & 13.04 & 0.91 & 0.93 & $0.087,0.082$ & 0.05 & - & DSCT \\
\hline V6 & - & $6^{\mathrm{h}} 02^{\mathrm{m}} 38,27^{\mathrm{s}}$ & $+49^{\circ} 53^{\prime} 04,7^{\prime \prime}$ & 14.47 & 0.39 & 1.09 & $1.1732,0.12936$ & 0.23 & 2452640.6072 & EA+DSCT: \\
\hline
\end{tabular}

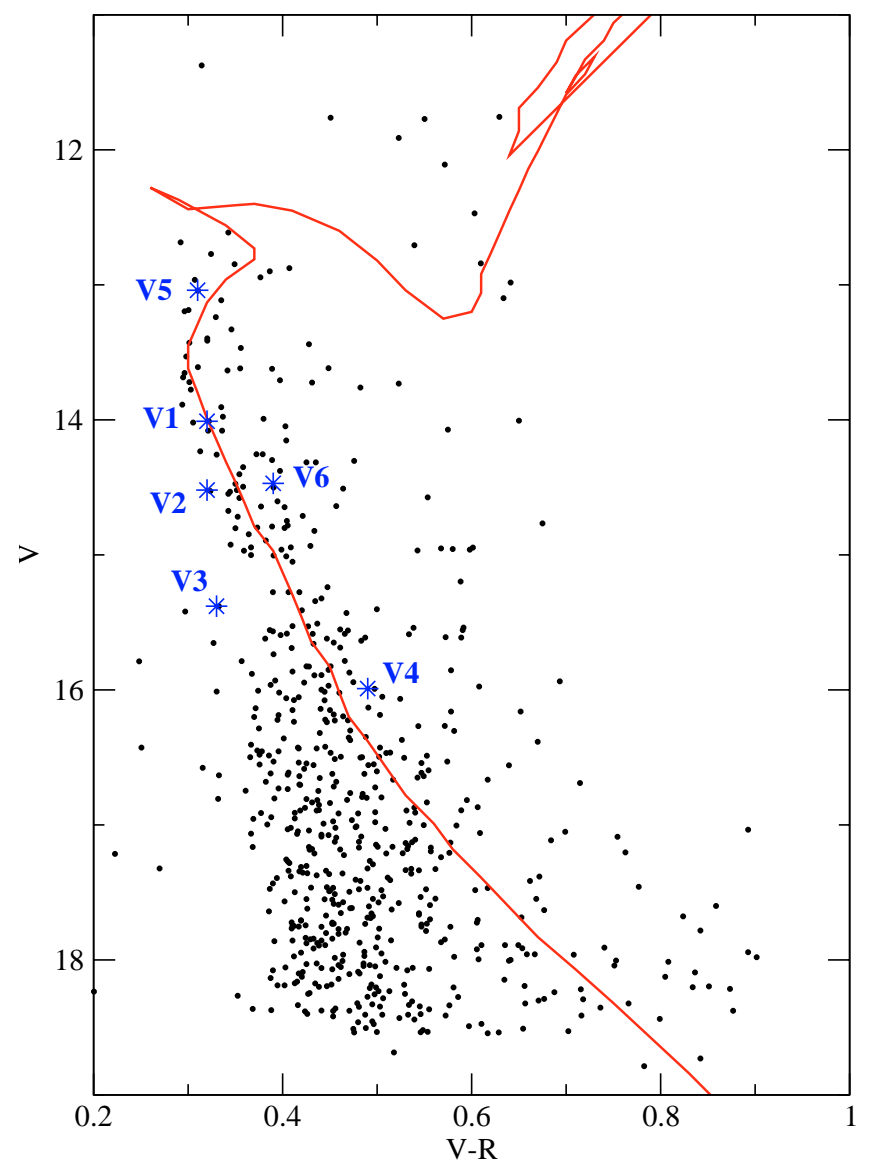

Fig. 10. Variable stars on the colour-magnitude diagram excluding foreground stars (except V6).

component. The period ratio $P_{\text {orb }} / P_{\text {pul }}=9.07$ may indicate a resonance between the orbital motion and pulsation.

We summarize the basic data of the variable stars in Table 3.

Acknowledgements. This work has been supported by the FKFP Grant 0010/2001, OTKA Grants \#F043203, \#T032258 and the Australian Research Council. Astronomy at the Wise Observatory is supported by grants from the Israeli Academy of Sciences. The NASA ADS Abstract Service was used to access data and references. This research has made use of the SIMBAD database, operated at CDSStrasbourg, France.

\section{References}

Arentoft, T., Sterken, C., Knudsen, M. R., et al. 2001, A\&A, 380, 599 Bertelli, G., Bressan, A., Chiosi, C., et al. 1994, A\&AS, 106, 275 Bessell, M. S. 1990, PASP, 102, 1181

Breger, M. 1972, ApJ, 176, 373

Breger, M., Stich, J., Garrido, R., et al. 1993, A\&A, 271, 482

Chevalier, C., \& Ilovaisky, S. A. 1991, A\&AS, 90, 225

Choo, K. J., Kim, S.-L., Yoon, T. S., et al. 2003, A\&A, 399, 99

Cox, A. N. (ed.) 2000, Allen's Astrophysical Quantities, 4th ed. (New York: AIP Press; Springer)

Cuffey, J. 1943, ApJ, 97, 93

Cutispoto, G., Messina, S., \& Rodonò, M. 2003, A\&A, 400, 659

Frandsen, S., \& Arentoft, T. 1998, A\&A, 333, 524

Gamarova, A. Yu., Mkrtichian, D. E., \& Kusakin, A. V. 2000, IBVS, No. 4837

Gilliland, R. L., \& Brown, T. M. 1988, PASP, 100, 754

Handler, G. 1999, MNRAS, 309, L19

Kim, S.-L., Chun, M.-Y., Park, B.-G., et al. 2001, A\&A, 371, 571

Kim, S.-L., Lee, J. W., Youn, J.-H., et al. 2002a, A\&A, 391, 213

Kim, S.-L., Kwon, S.-G., Youn, J.-H., et al. 2002b, IBVS, No. 5314

Kim, S.-L., Lee, J. W., Kwon, S.-G., et al. 2002c, IBVS, No. 5325

Kim, S.-L., Lee, J. W., Kwon, S.-G., et al. 2003, A\&A, 405, 231

Kiss, L. L., Szabó, M. Gy., Sziládi, K., et al. 2001, A\&A, 376, 561

Lyngå, G. 1987, Fifth catalogue of cluster parameters, Strasbourg

Mkrtichian, D. E., Kusakin, A. V., Gamarova, A. Yu., et al. 2002, ASP Conf. Ser., 256, 96

Monet, D. G., Levine, S. E., Canzian, B., et al. 2003, AJ, 125, 984

Ohshima, O., Narusawa, S.-Y., Akazawa, H., et al. 2001, AJ, 122, 418

Reed, M. D., et al. 2002, Radial and Nonradial Pulsations as Probes of Stellar Physics, ASP Conf. Ser., 259, 368

Rieke, G. H., \& Lebofsky, M. J. 1985, ApJ, 288, 618

Rodríguez, E., \& Breger, M. 2001, A\&A, 366, 178

Rodríguez, E., Claret, A., Sedano, J. L., et al. 1998, A\&A, 340, 196

Rucinski, S. M., Kaluzny, J., \& Hilditch, R. W. 1996, MNRAS, 282, 705

Space Telescope Science Institute (STSci) 2002, The Guide Star Catalog, Version 2.2

Sperl, M. 1998, Comm. Astr. Seis., 111

Stellingwerf, R. F. 1978, ApJ, 221, 661

Thompson, I. B., Kaluzny, J., Pych, W., et al. 2001, AJ, 121, 3089

Willems, B., \& Aerts, C. 2002, A\&A, 384, 441 